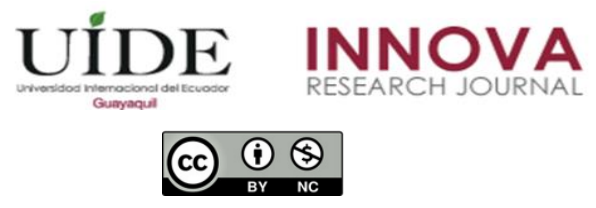

INNOVA Research Journal, ISSN 2477-9024

(Septiembre-Diciembre 2019). Vol. 4, No.3.1 pp. 1-17

DOI: https://doi.org/10.33890/innova.v4.n3.1.2019.1060

URL: http://revistas.uide.edu.ec/index.php/innova/index

Correo: innova@uide.edu.ec

\title{
Influencia de la administración del capital de trabajo en la rentabilidad empresarial
}

\section{Influence of working capital management on business profitability}

\author{
Gabriela Duque Espinoza \\ Orlando Espinoza Flores \\ Karla González Soto \\ Adrián Sigüencia Muñoz \\ Universidad del Azuay, Ecuador
}

Autor para correspondencia: gduque@uazuay.edu.ec; oespinoza@uazuay.edu.ec; kngonzalez@uazuay.edu.ec; asiguencia@uazuay.edu.ec.

Fecha de recepción: 12 de julio de 2019 - Fecha de aceptación: 24 de septiembre de 2019

\section{Resumen}

El capital de trabajo es relevante en la administración empresarial, pues proporciona a las firmas los recursos necesarios para llevar a cabo sus operaciones de forma regular, buscando una gestión eficiente que ayude a generar mayores niveles de rentabilidad. La presente investigación tiene por objeto determinar la eficiencia en la administración del capital de trabajo y su relación con la rentabilidad en las empresas del sector textil del Ecuador, y su evolución en el tiempo. Mediante una metodología de análisis cuantitativo, a través de datos de panel, se estudia la relación entre la eficiencia en la administración del capital de trabajo y la rentabilidad, de acuerdo con el nivel de actividad de 425 sociedades. Se evidencia que las firmas que utilizaron de manera más eficiente su capital de trabajo para la generación de ventas fueron las pequeñas y micro empresas. Las empresas pequeñas evidencian una relación positiva entre la variable de estudio y el ROA, lo que demuestra que tener una mayor eficiencia en la administración de capital de trabajo permite a las firmas obtener mayores niveles de rentabilidad.

Palabras Claves: capital de trabajo; rentabilidad; eficiencia empresarial; análisis sectorial; nivel de actividad

\begin{abstract}
Working capital is relevant in business management, since it provides firms with the necessary resources to carry out their regular operations, seeking efficient management that helps generate higher levels of profitability. The purpose of this research is to determine the efficiency in the management of working capital and its relationship with profitability in companies of the textile business sector of Ecuador, and their evolution over time. Through a quantitative analysis methodology, by means of panel data, the relationship between efficiency in the management of working capital and profitability is studied, according to size of 425 companies. It is evident that the firms that used their working capital more efficiently to generate sales were the small companies and microenterprises. Small companies show a positive relationship between the study
\end{abstract}


variable and ROA, which shows that having greater efficiency in the management of working capital allows firms to obtain higher levels of profitability.

Key words: working capital; business profitability; business efficiency; sectorial analysis; firm size

\section{Introducción}

Una de las principales problemáticas que enfrenta el sector empresarial ecuatoriano es la supervivencia de las firmas a lo largo del tiempo. De acuerdo con el informe para Ecuador del año 2017 elaborado por el Global Entrepreneurship Monitor GEM, (2017) el país se destaca por presentar el mayor índice de Actividad Emprendedora Temprana ${ }^{1}$ de la región de Sudamérica, 29,6\%; sin embargo, un 6,6 \% de estos nuevos emprendimientos no superan el primer año de actividades; siendo la razón más importante para el cese de actividades, de acuerdo al informe, los problemas para conseguir el financiamiento necesario para sus actividades, un $27 \%$ de negocios nuevos y un $25 \%$ de negocios establecidos cerraron por este motivo. Dentro de esta problemática, se enmarca la administración de recursos y financiamiento en el corto plazo, como un factor diferencial en la administración de empresas nuevas y establecidas con anterioridad.

El capital de trabajo es un factor relevante en la administración empresarial, puesto que proporciona a las firmas los recursos necesarios para llevar a cabo sus operaciones regulares de forma ininterrumpida, mediante el financiamiento de las inversiones de corto plazo. Según Harsh, (2014) una gestión eficiente del capital de trabajo permite a la empresa aprovechar efectivamente sus recursos para conseguir mayores niveles de ingresos con menores inversiones en activos corrientes, y reducir sus necesidades operativas de fondos; además de, adquirir la capacidad de enfrentar épocas de crisis sin la necesidad de afectar sus operaciones; por esta razón, es importante evaluar la gestión realizada por las empresas en cuanto a su capital de trabajo y cuál es su influencia sobre los resultados económicos.

El sector industrial sujeto de análisis es el de fabricación de prendas de vestir, excepto prendas de piel, el mismo que para el año 2017 obtuvo una producción de 328,77 millones de dólares; adicionalmente, este sector se caracteriza por el uso intensivo de mano de obra dada su naturaleza manufacturera; motivo por el cual, en el mismo año generó 8.411 plazas de empleo. (Superintendencia de Compañías Valores y Seguros del Ecuador, 2018).

El presente trabajo pretende esclarecer la relación existente entre la gestión del capital de trabajo y la rentabilidad, para lo cual se evalúa no únicamente la inversión en capital de trabajo, como lo hacen Ajay \& Gumbochuma, (2015), Mulyono, Djumahir, \& Ratnawati (2018), Debabrata (2018), y Zalaghi, Godini, \& Mansouri (2019); sino la gestión eficiente de la misma, tomando como referencia el trabajo realizado por Harsh, (2014). Partiendo de información financiera, se calcularon dos índices: rendimiento para la gestión del capital e índice de

1 Actividad Emprendedora Temprana - TEA: Porcentaje de la población adulta (entre 18 años y 64 años de edad) que está en el proceso de iniciar un negocio (emprendedor naciente), o ya es dueño o administrador de un negocio nuevo que ha estado en marcha durante menos de 42 meses. Este indicador se puede enriquecer con información relacionada con la motivación (oportunidad vs. necesidad), inclusión (género, edad), impacto (crecimiento del negocio en términos de generación de empleo, innovación, y sector industrial) e industria (sectores) (Global Entrepreneurship Monitor, 2017). 
utilización, con los cuales se estableció la eficiencia en la administración del capital de trabajo por parte de las compañías.

En una segunda parte, se realiza el análisis del índice de eficiencia de las empresas en base a sus niveles de actividad y su comportamiento en los años de estudio, evidenciando que a menor tamaño el índice de eficiencia de capital de trabajo es superior.

Finalmente, se determinó la relación que existe entre algunas variables financieras y la eficiencia en la administración del capital de trabajo. Las empresas dedicadas a la manufactura textil administraron adecuadamente su capital de trabajo con crecimientos en ventas más que proporcionales respecto al de la inversión en sus activos corrientes.

\section{Revisión de la literatura}

La relación entre la eficiencia en la administración del capital de trabajo y la rentabilidad ha sido explorada por autores como García \& Martínez (2007), Raheman \& Nasr (2007), Gill, Nahum, \& Mathur (2010), Debabrata (2018), sin embargo, no existe un consenso general respecto a la influencia que tiene la gestión del capital de trabajo sobre la rentabilidad de una empresa. En la revisión bibliográfica realizada, no se han podido encontrar estudios respecto a este tema en el Ecuador, por lo que el presente estudio es un tema de gran interés investigativo.

Como primer acercamiento a esta relación, es ineludible precisar algunos conceptos financieros. El término capital de trabajo es definido como "aquellos activos corrientes, que representan la parte de la inversión que circula de una forma a otra en la conducción ordinaria del negocio" (Gitman, 2007, p. 511). Un segundo concepto describe al capital de trabajo como "la inversión de una empresa en activos a corto plazo: efectivo, valores negociables, inventarios y cuentas por cobrar" (Besley \& Brighan, 2009, p. 554). Finalmente, "El término capital de trabajo se refiere a los activos de corto plazo de una empresa, como el inventario, y los pasivos de corto plazo, como dinero adeudado a los proveedores" (Ross, Westerfield, \& Jordan, 2010, p. 4).

Diferentes estudios han ampliado el concepto de capital de trabajo en función de su capacidad para crear valor a la empresa y a los inversionistas, asumiendo que la gestión de capital de trabajo afecta directamente a la liquidez y al resultado de la empresa (Raheman \& Nasr, 2007). En momentos de incertidumbre, tener una gestión eficaz de capital de trabajo puede ser crucial para la supervivencia de la empresa, especialmente para las pequeñas empresas (Padachi, 2006). Durante años, la gestión de capital de trabajo ha sido olvidada por los gestores debido al excesivo esfuerzo requerido en la modificación de las políticas a corto plazo, siendo lo común acudir al financiamiento a largo plazo cuando hacía falta financiamiento (Serrasqueiro, 2014), subestimando la importancia en la gestión del corto plazo.

Dada la naturaleza de incertidumbre de los ingresos en una empresa y la certeza de los pasivos en relación a su valor y fecha de vencimiento, resulta de importancia la eficiencia con la que las empresas transforman los activos corrientes en dinero para cubrir sus pasivos corrientes, y con esto generar una determinada rentabilidad. Esta transformación se lleva a cabo a través del Ciclo de Conversión de Efectivo, el cual evalúa el tiempo transcurrido desde que la empresa 
inicia la producción y compra bienes y servicios, hasta que cobra el valor proveniente de su venta, y con estos recursos paga las obligaciones en las que incurrió para producirlos o adquirirlos.

La rentabilidad está definida como "la relación entre los ingresos y los costos generados por el uso de los activos de la empresa (tanto corrientes como fijos) en actividades productivas" (Gitman, 2007, p. 512). La rentabilidad pudiera verse afectada por la gestión de los activos corrientes de la empresa, la cual a su vez depende de la administración del capital de trabajo; por lo tanto, resulta importante analizar la existencia de una relación entre la rentabilidad obtenida y la administración del capital de trabajo.

Sobre la relación de la administración de capital de trabajo y la rentabilidad de las empresas, los estudios evidencian tres tendencias claramente diferenciadas; la primera manifiesta que existe una relación inversa entre la inversión en capital de trabajo y la rentabilidad, apoyada por estudios empíricos llevados a cabo por autores como: Raheman \& Nasr (2007), quienes a través del análisis del comportamiento de 94 empresas listadas en la bolsa de valores de Pakistán, durante un período de 6 años, relacionan la rentabilidad operativa, con el período promedio de cobro, período promedio de inventario, período promedio de pago, ciclo de conversión de efectivo y razón corriente, encontrando una relación inversamente proporcional entre la rentabilidad y el capital de trabajo.

Una investigación similar fue llevada a cabo por García \& Martínez (2007) con datos de 8.872 pequeñas y medianas empresas españolas durante los años 1996 - 2002, utilizando la rentabilidad sobre activos (ROA) y variables independientes similares a las del estudio de Raheman \& Nasr, (2007), determinaron que existe una relación inversamente proporcional entre el capital de trabajo y la rentabilidad empresarial, y concluyeron que reducir los días de ciclo de conversión de efectivo influencia positivamente en la rentabilidad. Enow \& Brijlal (2014) obtienen los mismos resultados mediante igual estudio, con datos de 15 empresas, de la bolsa de valores de Johannesburgo, entre los años 2008 al 2014, y recomiendan mantener una estrategia de financiamiento a corto plazo agresiva.

Un estudio de Lazaridis \& Tryfonidis (2006), complementado y extendido por Gill et al., (2010) con datos de 88 empresas que cotizan en la bolsa de valores de Nueva York, de 5 sectores industriales, llegan a la conclusión de que existe una influencia negativa del capital de trabajo sobre la rentabilidad de las empresas; por lo tanto, los gerentes pueden incrementar la rentabilidad de sus empresas reduciendo el período del ciclo de conversión de efectivo.

Jayarathne (2014) condujo un estudio de 20 empresas del sector manufacturero que cotizan en la bolsa de valores de Sri Lanka desde el 2008 hasta el 2012, en el cual obtuvo resultados concordantes con los anteriores estudios mencionados, estableciendo una relación inversamente proporcional entre el capital de trabajo y la rentabilidad, sugiriendo una política de financiamiento de corto plazo agresiva.

Jaramillo (2016) evaluó el comportamiento de 48 empresas del sector de distribución de químicos en Colombia durante los años 2008 al 2014, encontrando una relación negativa entre el 
capital de trabajo y la rentabilidad de la empresa; en donde, a menor duración del ciclo de conversión de efectivo, más rentables son las empresas.

Prafitri, Rachmina, \& Ahmad, (2017) a través de su estudio realizado con información sobre 6 compañías de aceite de palma registradas en la bolsa de valores de Indonesia durante los años 2009 a 2015; explicaron el comportamiento de la rentabilidad sobre la inversión (ROI) a través del ciclo de conversión de efectivo, razón corriente, razón de endeudamiento y razón de activos financieros fijos, concluyendo que el capital de trabajo tiene un efecto negativo sobre la rentabilidad y que, cuanto más eficiente sea la administración del capital de trabajo, medida por el ciclo de conversión de efectivo, mayor será la rentabilidad de la empresa.

Kusuma \& Dhiyaullatief (2018) evaluaron el comportamiento de 44 empresas del sector manufacturero que cotizan en el Índice LQ45 de la bolsa de valores de Indonesia, cuyos resultados indicaron que existe una relación inversamente proporcional entre el capital de trabajo y la rentabilidad sobre activos (ROA), por ende, una mayor rotación del capital de trabajo conducirá a la empresa a un mayor resultado.

Los estudios mencionados hasta ahora obtienen resultados concordantes y concluyen acerca de la existencia de una influencia negativa de la inversión en capital de trabajo sobre la rentabilidad de una empresa, lo cual podría deberse a una gestión ineficiente de las cuentas que componen el activo corriente que deriva en una sobre inversión de recursos respecto al nivel de actividad ordinario de la empresa; es decir, niveles altos de período promedio de rotación de inventarios y cuentas por cobrar; así como un período promedio de pago bajo, los cuales no impulsan un crecimiento de las ventas; situación que incrementa el costo de financiamiento de corto plazo, reduciendo los márgenes de utilidad y los resultados obtenidos.

Una segunda tendencia instaura una influencia positiva de la inversión en capital de trabajo sobre la rentabilidad y recomienda una estrategia conservadora de financiamiento a corto plazo; Adam, Quansah, \& Kawor (2017) investigaron la relación de la rentabilidad sobre el patrimonio frente al ciclo de conversión de efectivo, razón corriente y la razón activo corriente sobre activo total, con datos de 6 empresas manufactureras que cotizan en la bolsa de valores de Ghana, sus resultados indicaron que la política de inversión en activos corrientes se relaciona positivamente con la rentabilidad a largo plazo; de forma que, a medida que aumenta la inversión en capital de trabajo, lo hace proporcionalmente también la rentabilidad sobre el patrimonio.

En concordancia con esta tendencia, se encuentra también el estudio realizado por Debabrata (2018), con datos de 15 empresas que cotizan en la bolsa de valores de la India por el período 2013 - 2017, relacionando rentabilidad sobre inversión (ROI), rentabilidad sobre activos (ROA) y rentabilidad sobre patrimonio (ROE), con variables que reflejan la gestión de capital de trabajo; además de tener en cuenta el tamaño empresarial en su análisis, sus resultados mostraron que la inversión en capital de trabajo tiene un efecto positivo sobre la rentabilidad.

Las investigaciones que dan soporte a esta segunda tendencia concluyen acerca de la existencia de una influencia positiva de la inversión en capital de trabajo sobre la rentabilidad de las empresas; lo cual podría explicarse por un aprovechamiento efectivo de los recursos adicionales invertidos en capital de trabajo, el cual infiere positivamente en el nivel de ventas y 
genera una mayor utilidad; es decir, el incremento en los períodos promedio de inventario y de cobro, o la reducción del período promedio de pago, pueden llegar a generar un mayor nivel de ventas que no solo compensa el costo financiero de estos recursos invertidos, sino que incrementa el rendimiento generado por la empresa.

La tercera tendencia, entendida como la administración de varios componentes del capital de trabajo, sostiene que las firmas deben mantener una adecuada cantidad de capital de trabajo que permita el buen e ininterrumpido funcionamiento de una empresa, a la vez del cumplimiento de objetivos simultáneos como liquidez y rentabilidad (Harsh, 2014) Estudios como los realizados por Afeef, Takreem, \& Baloch, (2015) a 436 Compañías no financieras listadas en la Bolsa de Valores de Pakistán desde el 2003 hasta el 2008, mediante el análisis de medidas de eficiencia en la administración del capital de trabajo, muestran que el período promedio de cobro, está relacionado negativamente con el retorno sobre activos.

En apoyo a esta tendencia se encuentra el estudio llevado a cabo por Ray (2014), durante los años 2004 al 2013, utilizando las variables razón corriente, rotación de inventario, rotación de cuentas por cobrar, margen de utilidad, el capital de trabajo sobre las ventas y activos corrientes sobre activos totales, además del retorno sobre los activos. Sus resultados indican una relación entre la rentabilidad y la rotación de inventario.

Harsh, (2014), estudió 13 empresas manufactureras del sector salud de la India, respecto de las cuales se tomaron datos de 14 años. El estudio analiza el cambio en el nivel de las cuentas que componen el activo corriente y su relación con la variación de las ventas, para determinar si las firmas generaron un incremento más que proporcional de las ventas respecto al crecimiento de la inversión en activos corrientes, situación que indica una administración eficiente; y posteriormente la relacionan con la rentabilidad. Sus resultados indican que la gestión ineficiente del capital de trabajo no solo reduce la rentabilidad, sino que, puede conducir a una crisis financiera.

Como la evidencia empírica demuestra, no existe un consenso general acerca de cuál es la relación existente entre la administración del capital de trabajo y la rentabilidad, si bien gran parte de investigaciones apuntan hacia un efecto negativo del capital de trabajo sobre la rentabilidad, sugiriendo reducir en lo posible la duración del ciclo de conversión de efectivo, en búsqueda de la eficiencia y llevar a cabo una política de financiamiento de corto plazo agresiva, existen también estudios que manifiestan exactamente lo contrario, y consideran la existencia de un efecto positivo del capital de trabajo sobre la rentabilidad, por lo que para obtener mayor rentabilidad es necesario extender el ciclo de conversión de efectivo en lo posible y mantener una política de financiamiento de corto plazo conservadora. Ante tal situación, el presente estudio contribuirá a esclarecer el comportamiento de las empresas en el Ecuador, en cuanto a la gestión del capital de trabajo y su efecto en la rentabilidad.

\section{Metodología}

El estudio mantiene un enfoque cuantitativo, mediante el análisis de 425 empresas del sector Textil reguladas bajo la Superintendencia de Compañías, Valores y Seguros del Ecuador, con código CIIU1410 Fabricación de prendas de vestir, excepto prendas de piel. La investigación

Esta obra se comparte bajo la licencia Creative Common Atribución-No Comercial 4.0 International (CC BY-NC 4.0) 
es de corte longitudinal, a través de datos de panel, debido a que se obtuvieron datos del periodo 2014 al 2017 para las empresas del sector mencionado.

Las variables utilizadas en el estudio son las distintas cuentas que componen el activo corriente de las empresas, y los ingresos por ventas. En el caso de la rotación de los inventarios, la rotación de cuentas por cobrar, el rendimiento sobre la inversión y el rendimiento sobre los activos, se han considerado los ratios financieros obtenidos de la información contable de los estados de resultados y de situación financiera reportados. Se utilizó la información de aquellas empresas que presentan valores de ventas durante el periodo de análisis, con la finalidad de calcular el índice de eficiencia en la administración del capital de trabajo.

La clasificación de las empresas se realizó conforme lo establece el Código Orgánico de la Producción, Comercio e Inversiones, dividiéndolas en microempresa, pequeña, mediana y grande; considerando para ello los parámetros de montos de ingresos y número de trabajadores.

Para el procesamiento de la información, los valores atípicos fueron tratados mediante el criterio de Chauvenet ${ }^{2}$. Se aplicó un análisis ANOVA para determinar las diferencias estadísticamente significativas de las medias promedio del sector, en base a su clasificación por tamaño.

Harsh, (2014) proponen un modelo para determinar la eficiencia en la administración del capital de trabajo de las firmas, medido a través de dos variables, el mismo que fue considerado para el presente estudio.

La primera se basa en el Índice de rendimiento para la gestión del capital (PI), el cual mide la relación entre el aumento de las ventas y el aumento del activo corriente en términos de proporción. Cuando el aumento proporcional de las ventas es mayor, pues el índice será superior a 1, lo que indica una gestión eficiente (Harsh, 2014)

$$
\mathrm{PI}_{(W C M)}=\frac{\operatorname{Is} \sum_{i=1}^{n} W_{i(t-1)} / W_{i t}}{N}
$$

Dónde: $\mathrm{I}_{\mathrm{s}}=$ Índice de ventas $\mathrm{S}_{t} / \mathrm{S}_{t-1}, \mathrm{~W}_{\mathrm{i}}=$ Cuentas que componen el activo corriente, $\mathrm{N}$ $=$ Número de cuentas que componen el activo corriente, $\mathrm{I}=1,2,3 \ldots \mathrm{N}$.

La segunda variable mide la capacidad de generar ventas por medio de la utilización de los activos corrientes, considerando su valor total. La variable considera el cambio de un año en relación al anterior, esperando un valor superior a 1, a lo cual se denomina Índice de utilización (UI) (Harsh, 2014).

$$
\mathrm{UI}_{(\mathrm{WCM})}=\frac{\mathrm{A}_{(t-1)}}{\mathrm{A}_{t}}(2)
$$

${ }^{2}$ El criterio de Chauvenet es un método que consiste en determinar si un dato experimental de un conjunto de mediciones es probable que sea un dato atípico. Se establecen límites inferior y superior en función de la desviación típica de la muestra de datos, y todas las medidas que superen esos límites deben ser rechazadas. 
Dónde: $\mathrm{A}=$ Activo corriente / Ventas.

La eficiencia en la administración del capital de trabajo es medida en base a los valores combinados de la media del rendimiento para la gestión del capital y la utilización del mismo, siendo esta la métrica base aplicada al sector textil sobre la cual las empresas que forman parte del mismo pueden medir su desempeño individual.

$$
\mathrm{EI}_{(\mathrm{WCM})}=\mathrm{PI}_{(\mathrm{WCM})} * \mathrm{UI}_{(\mathrm{WCM})}(3)
$$

Se analizó la tendencia del índice de eficiencia, considerando los cuatro años de estudio, según el tamaño de las firmas, para poder evaluar su comportamiento en el tiempo. Finalmente, se analizaron los valores obtenidos de eficiencia en la administración del capital de trabajo y su relación con la rotación del inventario ${ }^{3}(\mathrm{RI})$, rotación de cuentas por $\operatorname{cobrar}^{4}(\mathrm{RCOB})$, rendimiento sobre activos $^{5}$ (ROA) y rendimiento sobre el patrimonio ${ }^{6}$ (ROE) considerando los diferentes cambios de actividad empresarial.

\section{Resultados y Discusión}

Se presenta un análisis de la eficiencia en la administración del capital de trabajo de las empresas ecuatorianas del sector textil, medida a través del aprovechamiento y uso de las cuentas que conforman el activo corriente en relación con los cambios en las ventas en el periodo de estudio. La tabla 1 muestra algunas medidas de tendencia central y dispersión de las empresas dedicadas a la producción textil, mediante el análisis de las variables que forman parte de la presente investigación.

3 "La rotación de inventarios refleja la velocidad con la que la empresa desplaza su inventario desde las materias primas, a través de la producción, hasta los productos terminados y al cliente como una venta".

$\mathrm{RI}=\frac{\text { Costo de los bienes vendidos }}{\text { Inventario }}$ (Gitman, 2007, p. 49)

${ }^{4}$ La rotación de cuentas por pagar "se usa para evaluar la capacidad de una empresa para cobrar sus ventas a crédito de manera oportuna". RCOB = $\frac{\text { Cuentas por cobrar }}{\text { Ventas a crédito }}$ (Besley \& Brighan, 2009, p. 55).

5 "El Rendimiento sobre Activos es una medida de la utilidad por dólar de activos. Se puede definir en varias formas. ROA $=\frac{\text { Utilidad neta }}{\text { Total de Activos }} "$ (Ross et Al., 2010, p. 62)

6 "El Rendimiento sobre Patrimonio es una medida de cómo les fue a los accionistas durante el año. Debido a que la meta consiste en beneficiar a los accionistas, el ROE, en términos contables, es la verdadera medida del desempeño del renglón básico". $\mathrm{ROE}=\frac{\text { Utilidad Neta }}{\text { Capital contable total }}($ Ross, et Al. 2010, p. 62) 
Tabla 1.

Estadísticos Descriptivos

\begin{tabular}{|c|c|c|c|c|c|c|c|c|c|c|c|c|}
\hline & & & & & Ran & & Míni & & Máxi & & $\mathrm{Me}$ & Desviac \\
\hline & & & $\mathrm{N}$ & go & & mo & & mo & & dia & & ión estándar \\
\hline & EI & & 3 & & 59,1 & & - & & 59,03 & & 1,83 & 4,751 \\
\hline & & 05 & & 04 & & 0,068 & & 6 & & 8 & & \\
\hline & RO & & 4 & & 7,94 & & - & & 1,454 & & - & 0,443 \\
\hline A & & 25 & & 3 & & 6,489 & & & & 0,030 & & \\
\hline & RO & & 4 & & 12,5 & & - & & 8,019 & & 0,12 & 0,657 \\
\hline $\mathrm{E}$ & & 25 & & 34 & & 4,515 & & & & 9 & & \\
\hline & $\mathrm{RC}$ & & 2 & & 36,0 & & 0,000 & & 36,08 & & 3,29 & 4,880 \\
\hline PAG & & 88 & & 88 & & & & 8 & & 3 & & \\
\hline $\mathrm{COB}$ & $\mathrm{RC}$ & 92 & 2 & 00 & 31,1 & & 0,000 & 0 & 31,10 & 4 & 6,47 & 4,880 \\
\hline & $\mathrm{R}$ & & 2 & & 82,9 & & 0,000 & & 82,90 & & 9,11 & 12,940 \\
\hline INV & & 40 & & 04 & & & & 4 & & 8 & & \\
\hline
\end{tabular}

Fuente: Superintendencia de Compañías, Valores y Seguros.

Durante el periodo comprendido entre los años 2014 - 2017, el sector analizado presenta una media de indicador de eficiencia de 1,84, lo que indica que en promedio las empresas dedicadas a la manufactura textil administraron adecuadamente su capital de trabajo con crecimientos en ventas más que proporcionales respecto al de la inversión en sus activos corrientes; con respecto a los indicadores de actividad, las cuentas por cobrar en promedio se efectivizaron 6,474 veces en el año (56 días), las compañías pagan sus cuentas a proveedores con una media de 3,293 veces (111 días), lo cual denotaría un elevado poder de negociación con los proveedores, y por lo tanto, facilidad de obtener una fuente adicional de financiamiento a corto plazo. La rotación promedio es de 9,12 veces (40 días).

Analizando el tiempo promedio en que las empresas llevan a cabo su ciclo de conversión de efectivo, se determina que han alcanzado niveles de actividad eficientes demostrados por una recuperación más rápida de su efectivo en comparación con los plazos otorgados con proveedores.

Tal como lo evidencia la tabla 2, se realiza el cálculo del índice de eficiencia del capital de trabajo, la misma que contempla por una parte el desempeño promedio de los componentes del activo corriente, y por otra el uso del activo corriente como generador de ventas, considerando los niveles de actividad de las firmas.

Tabla 2.

Índice de Eficiencia en la administración del Capital de Trabajo.

\begin{tabular}{llll}
\hline Tamaño & *EI & PI & UI \\
\hline Microempresas & 2,593 & 2,018 & 1,285 \\
Pequeñas & 1,439 & 1,408 & 1,022 \\
Medianas & 1,009 & 1,077 & 0,937 \\
Grandes & 0,891 & 0,909 & 0,980 \\
\hline
\end{tabular}

Fuente: Superintendencia de Compañías, Valores y Seguros (2018)

Nota: Test ANOVA, nivel de significatividad menor al $5 \%$.

Nota: Test POST HOC Tukey, nivel de significatividad menor al 5\%.

Nota: EI=PI x UI 
El índice de rendimiento para la gestión de capital de trabajo (PI) para las microempresas, pequeñas y medianas es mayor a 1 , donde el incremento en las ventas es mayor en términos de proporción que el crecimiento individual de las cuentas que componen el activo corriente; mientras que, en el caso de las grandes compañías, las ventas no crecen en la misma proporción en relación a las cuentas del activo corriente. Estos resultados demuestran que las micro, pequeñas y medianas empresas aprovechan en mayor proporción el activo corriente en relación a la capacidad que tienen para generar ingresos.

A través de la obtención del índice de utilización de los activos (UI), se determinó que aquellos que utilizaron de manera más eficiente los activos para la generación de las ventas fueron las micro y pequeñas empresas, con índices de 1,285 y 1,022 respectivamente.

La combinación de los dos indicadores analizados anteriormente mide la eficiencia en la administración de capital de trabajo (EI), cuyo resultado demuestra que las microempresas y pequeñas son más eficientes en su administración de capital de trabajo lo cual denota un mejor uso de su activo corriente, que repercute en un mayor nivel de ingresos obtenidos por estas empresas.

La tabla 3 resume el análisis de eficiencia en la gestión de capital de trabajo considerando los niveles de actividad de las firmas debido a las diferencias significativas encontradas en la aplicación del test de medias; por lo que, analizarlas bajo este criterio y su evolución en el tiempo permite determinar su comportamiento durante el periodo de análisis.

\section{Tabla 3.}

Índice de eficiencia en la administración del capital de trabajo de las empresas del Sector Textil, según año y nivel de actividad.

\begin{tabular}{lllll}
\hline Año & Microempresas & Pequeñas & Medianas & Grandes \\
\hline 2014 & 0,79 & 1,51 & 1,31 & 1,08 \\
2015 & 2,08 & 1,61 & 1,09 & 0,74 \\
2016 & 4,03 & 1,17 & 0,90 & 0,96 \\
2017 & 6,32 & 2,47 & 0,98 & 1,05 \\
\hline
\end{tabular}

Fuente: Superintendencia de Compañìas, Valores y Seguros (2018)

Nota: Test ANOVA, nivel de significatividad menor al $1 \%$.

Se evidencia que las pequeñas empresas son eficientes en la administración de capital de trabajo y que esta eficiencia crece con los años, con excepción del año 2016 las microempresas pasan de ser ineficientes con un índice de 0,79 para el año 2014 a tener un indicador de 6,32 para el periodo 2017, mostrando altos niveles de eficiencia.

Durante los dos primeros años, las medianas empresas demuestran eficiencia en su operación; sin embargo, a partir del 2016 pasan a ser ineficientes, esto se debe a que la proporción de activos corrientes fue mayor en relación al crecimiento en el nivel de ventas. 
El análisis de correlación de las variables que sirven como base para el estudio, permiten determinar su influencia en el rendimiento de las empresas que conforman el sector analizado.

Tabla 4.

Análisis de correlación, microempresas

\begin{tabular}{|c|c|c|c|c|c|c|c|c|c|c|c|c|c|}
\hline & & & $\mathrm{Ut}$ & & $\mathrm{R}$ & & $\mathrm{R}$ & & $\mathrm{R}$ & & $\mathrm{R}$ & & $\mathrm{R}$ \\
\hline & & $\mathrm{i}$ & ilidad Neta & OA & & OE & & inv. & & c cob. & & c pag. & \\
\hline EI & & Corr & 0. & & 0 & & 0 & & - & & - & & 0 \\
\hline & elación & & 127 & .141 & & .054 & & 0.018 & & 0.190 & & .016 & \\
\hline & & Sig. & 0 . & & 0 & & 0 & & 0 & & 0 & & 0 \\
\hline & & & 368 & .318 & & .702 & & 925 & & .313 & & 932 & \\
\hline $\mathrm{Ut}$ & & Corr & 1 & & . & & - & & 0 & & 0 & & 0 \\
\hline ilidad neta & elación & & & $311^{* *}$ & & $.231^{* * *}$ & & . 168 & & .053 & & .149 & \\
\hline & & Sig. & & & 0 & & 0 & & 0 & & 0 & & 0 \\
\hline $\mathrm{R}$ & & Corr & & .000 & 1 & .003 & _ & 299 & 0 & 695 & - & .316 & 0 \\
\hline OA & elación & & & & & $.161^{*}$ & & 209 & & 0.037 & & .034 & \\
\hline & & Sig. & & & & & 0 & & 0 & & 0 & & 0 \\
\hline & & & & & & .037 & & 196 & & .787 & & .823 & \\
\hline $\mathrm{R}$ & & Corr & & & & & 1 & & 0 & & . & & 0 \\
\hline $\mathrm{OE}$ & elación & & & & & & & .023 & & $269^{*}$ & & .124 & \\
\hline & & Sig. & & & & & & & 0 & & 0 & & 0 \\
\hline & & & & & & & & .889 & & .043 & & .408 & \\
\hline R. & & Corr & & & & & & & 1 & & - & & . \\
\hline Inv. & elación & & & & & & & & & 0.010 & & $415^{*}$ & \\
\hline & & SIg. & & & & & & & & .964 & 0 & .039 & 0 \\
\hline
\end{tabular}

Fuente: Superintendencia de Compañías, Valores y Seguros. (2018)

Nota: ** Significancia estadística al $1 \%$ (bilateral).

Nota: * Significancia estadística al $5 \%$ (bilateral).

En el caso de las microempresas, conforme los resultados resumidos en la tabla 4, un incremento de la utilidad neta afecta de manera directa al ROA, con un nivel de confianza del 99\%. También se observa que existe una influencia positiva de la rotación de cuentas por cobrar sobre el ROE, que manifiesta que aquellas empresas que logran recuperar su cartera en menor tiempo, fueron más rentables, lo cual ratifica los resultados obtenidos por Debabrata (2018).

Tabla 5.

Análisis de correlación, pequeñas empresas

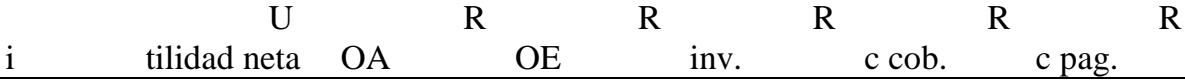




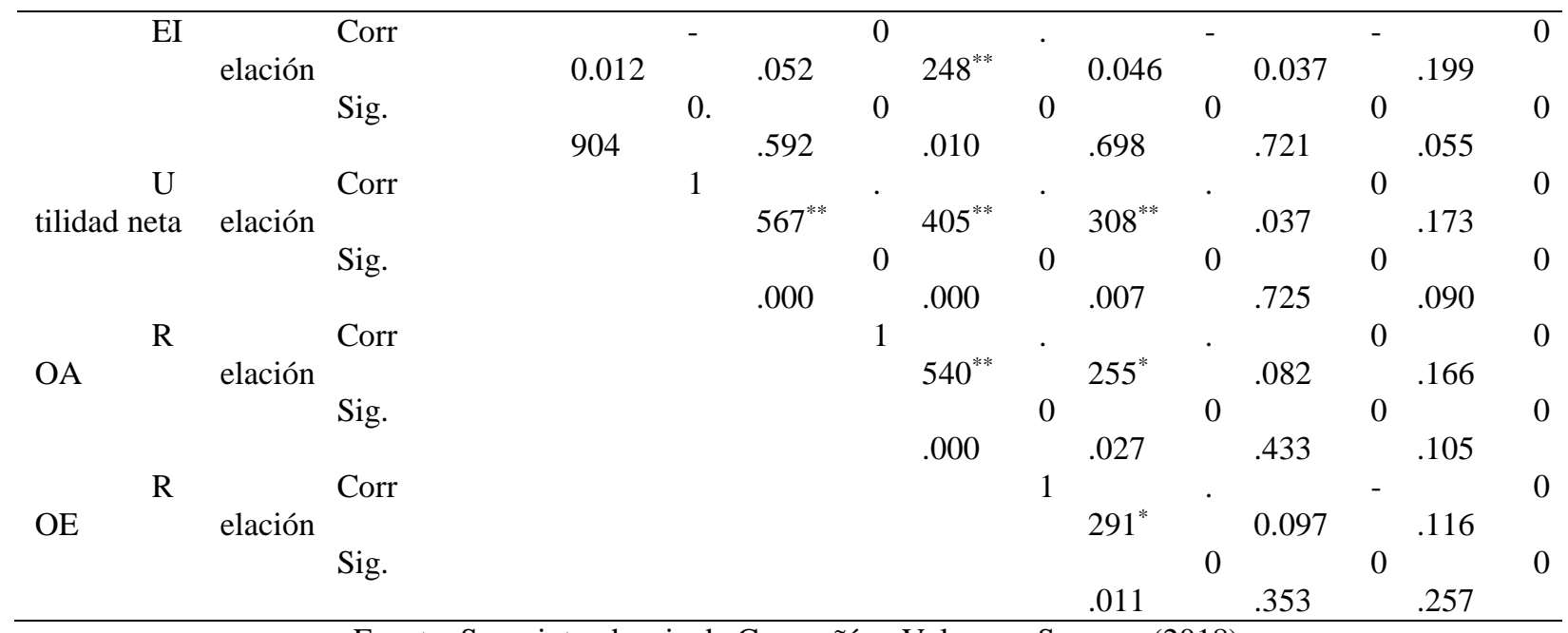

Fuente: Superintendencia de Compañías, Valores y Seguros (2018).

Nota: ** Significancia estadística al $1 \%$ (bilateral).

Nota: * Significancia estadística al 5\% (bilateral).

Para las firmas pequeñas del sector textil, cuyos resultados constan en la tabla 5, la relación que existe entre la utilidad neta y la rentabilidad con relación a sus activos y patrimonio es positiva; es decir, que aquellas empresas que presentan una mayor utilidad neta tienen una mayor rentabilidad proporcional a sus activos y patrimonio. Con relación a la gestión de inventarios, se refleja que aquellas empresas que mantienen mejores índices de actividad, obtuvieron un incremento en la rentabilidad sobre activos, patrimonio y utilidad, debido a que, mantener una baja rotación de inventarios, implica mayores costes de mantenimiento y almacenamiento que afectan la rentabilidad, similar a los resultados obtenidos por Raheman $\&$ Nasr, (2007) García \& Martínez, (2007). En cuanto a la variable de estudio, se evidencia que existe una relación positiva y significativa entre la eficiencia en la administración de capital de trabajo y el ROE, lo que muestra que a mayor eficiencia mayor será el rendimiento sobre el patrimonio empresarial. 
Tabla 6.

Análisis de correlación, medianas empresas

\begin{tabular}{|c|c|c|c|c|c|c|c|c|c|c|c|c|c|}
\hline & & & $\mathrm{U}$ & & $\mathrm{R}$ & & $\mathrm{R}$ & & $\mathrm{R}$ & & $\mathrm{R}$ & & $\mathrm{R}$ \\
\hline & & $\mathrm{i}$ & tilidad neta & $\mathrm{OA}$ & & $\mathrm{OE}$ & & inv. & & c cob. & & c pag. & \\
\hline & & Corr & 0. & & 0 & & 0 & & - & & - & & 0 \\
\hline FI & elación & & 031 & .043 & & .034 & & 0.047 & & 0.030 & & .062 & \\
\hline Li & & Sig. & 0. & & 0 & & 0 & & 0 & & 0 & & 0 \\
\hline & & & 744 & .649 & & .719 & & .651 & & .754 & & .513 & \\
\hline & & Corr & 1 & & . & & . & & 0 & & 0 & & 0 \\
\hline $\mathrm{U}$ & elación & & & $688^{* *}$ & & $591^{* *}$ & & .083 & & .089 & & .075 & \\
\hline tilidad neta & & Sig. & & & 0 & & 0 & & 0 & & 0 & & 0 \\
\hline & & & & .000 & & .000 & & .424 & & .347 & & .429 & \\
\hline & & Corr & & & 1 & & . & & $\cdot$ & & • & & 0 \\
\hline $\mathrm{R}$ & elación & & & & & $913^{* *}$ & & $235^{*}$ & & $209^{*}$ & & .096 & \\
\hline $\mathrm{OA}$ & & Sig. & & & & & 0 & & 0 & & 0 & & 0 \\
\hline & & & & & & . 000 & & .022 & & . 026 & & .310 & \\
\hline & & Corr & & & & & 1 & & . & & 0 & & 0 \\
\hline $\mathrm{R}$ & elación & & & & & & & $381^{* *}$ & & .150 & & . 093 & \\
\hline $\mathrm{OE}$ & & Sig. & & & & & & & 0 & & 0 & & 0 \\
\hline & & & & & & & & . 000 & & .113 & & .326 & \\
\hline & & Corr & & & & & & & 1 & & - & & \\
\hline $\mathrm{R}$ & elación & & & & & & & & & 0.127 & & $354^{* *}$ & \\
\hline inv. & & Sig. & & & & & & & & & 0 & & 0 \\
\hline & & & & & & & & & & .225 & & .000 & \\
\hline
\end{tabular}

Fuente: Superintendencia de Compañías, Valores y Seguros.

Nota: ** Significancia estadística al $1 \%$ (bilateral).

Nota: * Significancia estadística al $5 \%$ (bilateral).

En las empresas medianas, de acuerdo con los resultados expresados en la tabla 6, la utilidad neta influye en forma positiva sobre la rentabilidad medida en proporción de activos y patrimonio. En cuanto al nivel de actividad medido como la rotación de inventario, se muestra que mientras más rápido rotan los inventarios se obtendrán mayores niveles de rentabilidad sobre activos y sobre patrimonio. A una conclusión similar llegaron Debabrata (2018), Kusuma \& Dhiyaullatief, (2018). La eficiencia en la realización de la cartera en este grupo de empresas, influyó de manera positiva en la rentabilidad sobre activos. 
Tabla 7.

Análisis de correlación, grandes empresas

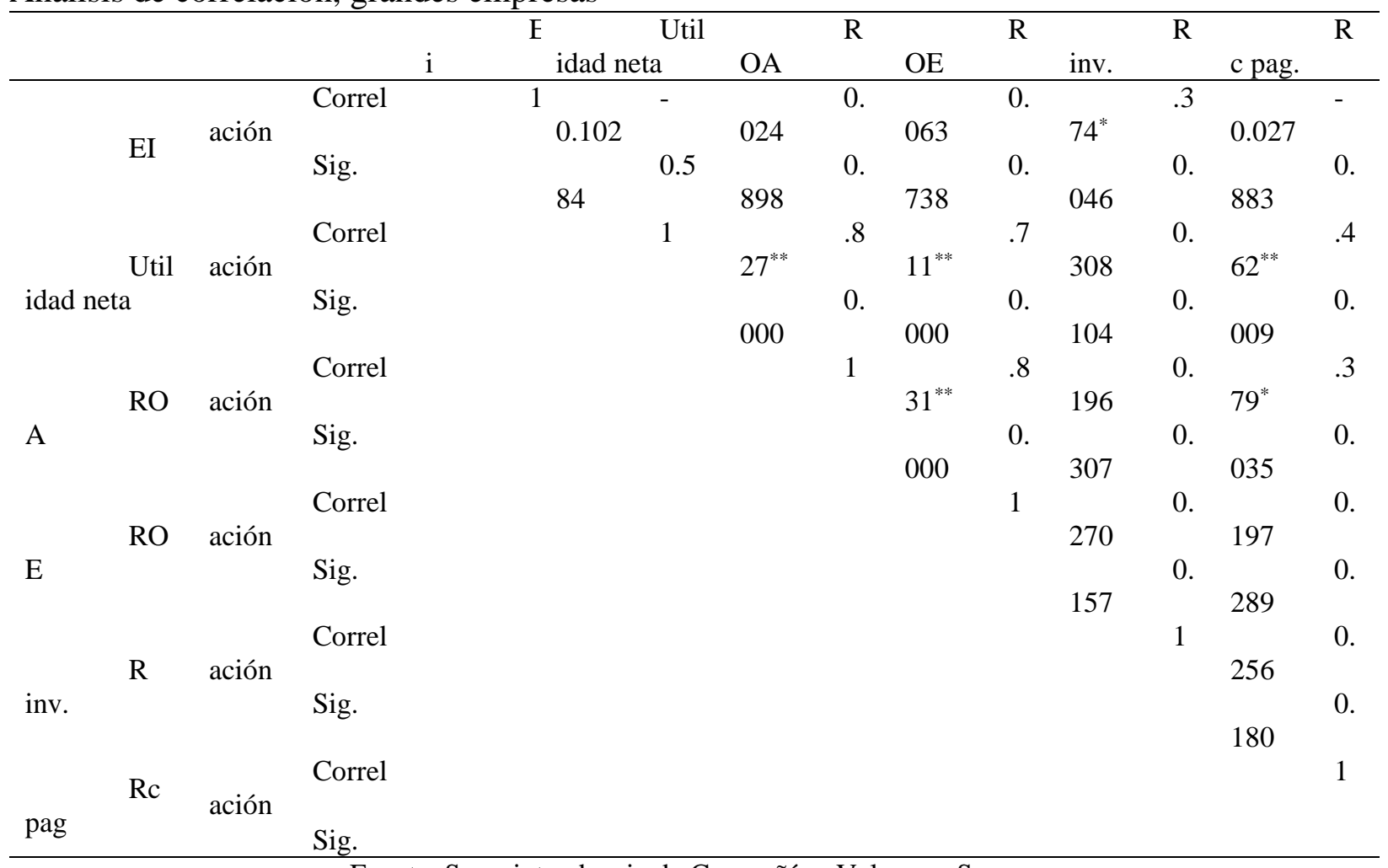

Fuente: Superintendencia de Compañías, Valores y Seguros.

Nota: ** Significancia estadística al $1 \%$ (bilateral).

Nota: * Significancia estadística al 5\% (bilateral).

Los resultados de la tabla 7 relacionados con las grandes empresas, evidencia que el índice de eficiencia en la administración de capital de trabajo se ve incrementado de forma proporcional a medida que rotan sus inventarios. Además, se demuestra que cumplir de forma eficiente con el pago a proveedores aumenta la rentabilidad sobre activos y los niveles de utilidad neta.

Los resultados del presente estudio, ratifican lo obtenido por Raheman \& Nasr (2007), Enow \& Brijlal (2014), García \& Martínez (2007), Lazaridis \& Tryfonidis (2006), Jayarathne (2014), Jaramillo (2016), ya que en el caso de las pequeñas y medianas empresas del sector textil en el Ecuador, la relación es positiva entre la rotación de inventarios y la rentabilidad, evidenciando que una mayor eficiencia en la gestión del inventario influye positivamente en los resultados empresariales.

La eficiencia en la administración del capital de trabajo y su relación con la rentabilidad, se ve evidenciada solo en las pequeñas empresas, en concordancia con los estudios de Harsh, (2014), Afeef et al., (2015) y Ray (2014). Esto demuestra que a mayor eficiencia se garantizan mayores niveles de rentabilidad empresarial. 


\section{Conclusiones}

La eficiencia en la administración de capital de trabajo se ve influenciada por el nivel de actividad de las empresas, debido a que un incremento en el activo corriente no afecta a la generación de ventas con la misma intensidad en las grandes empresas que en las micro, pequeñas o medianas empresas, lo que sugiere que la estructura organizacional puede constituirse en un factor relevante para mejorar la utilización del capital de trabajo.

Mediante la aplicación del modelo propuesto por Harsh, (2014), aplicado en las empresas del sector textil en el Ecuador, se concluye que las micro y pequeñas empresas muestran los mayores niveles de eficiencia en la administración de capital de trabajo, impulsado mayoritariamente por el rendimiento para la gestión del capital (PI); es decir, debido a que el aumento de las ventas es mayor al incremento del activo corriente medido en términos de proporción.

Las micro y pequeñas empresas en el tiempo han mejorado su índice de eficiencia en la administración del capital de trabajo, a diferencia de las medianas empresas que han ido en deterioro.

Se pudo evidenciar que, en las pequeñas empresas del Sector Textil una mayor eficiencia en la administración de capital de trabajo influye de manera positiva en el rendimiento sobre la inversión en activos, lo que promueve que las empresas deben centrar sus esfuerzos en una eficiente gestión de las partidas de corto plazo son el fin de incrementar sus resultados empresariales.

La presente investigación resulta de utilidad para visualizar el comportamiento de las empresas del Sector Textil según sus niveles de actividad en lo relacionado al capital de trabajo y la rentabilidad, y proporciona información que aporta a una mejor toma de dediciones, basada en estrategias que incrementen el valor de una firma.

Como futuras líneas de investigación, se sugiere el análisis de otros sectores económicos, para conocer su realidad y que permitan realizar comparación entre las distintas ramas de la economía.

\section{Bibliografía}

Adam, A., Quansah, E., \& Kawor, S. (2017). Working Capital Management policies and returns of listed manifacturing firms in Ghana. Scientific Annals of Economics and Business, 64(2), 255-269. https://doi.org/10.1515/saeb-2017-0017

Afeef, M., Takreem, K., \& Baloch, Q. (2015). Does Efficient Management of Working Capital have a Parallel Impact on the Profitability of Small and Large Firms? Journal of Humanities \& Social Sciences, 3(1), 1-18. Retrieved from https://search.proquest.com/docview/1815500288?accountid=36552

Ajay, G., \& Gumbochuma, I. (2015). Relationship between working capital management and profitability in JSE listed retail sector companies. Investment Management and Financial Innovations, 12(2), 127-135. 
Besley, S., \& Brighan, E. (2009). Fundamentos de Administración Financiera. Tampa.

Debabrata, J. (2018). Impact of Working Capital Management on Profitability of the Selected Listed FMCG Companies in India. International Research Journal of BUSINESS STUDIES, 11(1), 21-30. https://doi.org/https://doi.org/10.21632/irjbs.11.1.21-30

Enow, S., \& Brijlal, P. (2014). The effect of working capital management on profitability: The case of Small Medium and Micro Enterprises in South Africa. Journal of Accounting and Management, 4(2), 7-15.

García, P., \& Martínez, P. (2007). Effects of working capital management on SME profitability. International Journal of Managerial Finance, 3(2), 164-177. Retrieved from https://doi.org/10.1108/17439130710738718

GEM. (2017). Global Entrepreneuship Monitor-Ecuador 2017, 80.

Gill, A., Nahum, B., \& Mathur, N. (2010). The Relationship between Working Capital Management and Profitability: Evidence from the United States. Business and Economics Journal, 10(1), 1-9. Retrieved from http://astonjournals.com/bej

Gitman, L. (2007). Principios de Administración Financiera. San Diego.

Harsh, K. (2014). Efficient Management of Working Capital : a Study of Heathcare Sector in India. Management Strategies Journal, 25(3), 53-65. Retrieved from http://www.strategiimanageriale.ro/papers/140305.pdf

Jaramillo, S. (2016). Relación entre la gestión del capital de trabajo y la rentabilidad en la industria de distribución de químicos en Colombia. Revista Finanzas y Política Económica, 8(2), 327-347. https://doi.org/http://dx.doi.org/10.14718/revfinanzpolitecon.2016.8.2.6

Jayarathne, T. (2014). Impact of Working Capital Management on Profitability: Evidence From Listed Companies in Sri Lanka. In 3rd International Conference on Management and Economics (pp. 269-274).

Kusuma, H., \& Dhiyaullatief, A. (2018). Working Capital Management and Corporate Performance: Evidence from Indonesia. Journal of Management and Business Administration. Central Europe, 26(2), 76-88. https://doi.org/https://doi.org/10.7206/jmba.ce.2450-7814.229

Lazaridis, I., \& Tryfonidis, D. (2006). Relationship between Working Capital Management and Profitability of Listed Companies in the Athens Stock Exchange. Journal of Financial Management and Analysis, 19(1), 1-12. Retrieved from https://search.proquest.com/docview/215226709?accountid=36552

Mulyono, S., Djumahir, D., \& Ratnawati, K. (2018). The Effect of Capital Working Management on the Profitability. Jurnal Keuangan Dan Perbankan, 22(1), 94-102. https://doi.org/10.26905/jkdp.v22i1.1332

Padachi, K. (2006). Trends in working capital management and its impact on firms' performance: An analysis of Mauritian Small Manufacturing Firms. International Review of Business Research Papers, 2(2), 45-58.

Prafitri, T., Rachmina, D., \& Ahmad, T. (2017). The effect of working capital on the profitability of palm oil plantation companies. Indonesian Journal of Business and Entrepreneurship, 3(2), 111-120. https://doi.org/10.17358/IJBE.3.2.111

Raheman, A., \& Nasr, M. (2007). Working Capital Management And Profitability - Case Of Pakistani Firms. International Review of Business Research Papers, 3(1), 279-300.

Ray, K. (2014). Efficiency of Working Capital Management and Profitability: A Case of Hindalco. Review of Management, 4(1), 19-30. 
Ross, S., Westerfield, R., \& Jordan, B. (2010). Fundamentos de Finanzas Corporativas. Cambridge.

Serrasqueiro, J. (2014). Working Capital Management impact on Profitability.

Superintendencia de Compañías Valores y Seguros del Ecuador. (2018). Superintendencia de Compañías, Valores y Seguros del Ecuador. Retrieved from https://www.supercias.gob.ec/portalscvs/

Zalaghi, H., Godini, M., \& Mansouri, K. (2019). The Moderating Role of Firms Characteristics on the Relationship between Working Capital Management and Financial Performance. Advances in Mathematical Finance and Applications, 4(1), 71-88. https://doi.org/10.22034/amfa.2019.581878.1158 